\title{
вмј Global Health COVID-19 diagnostics: preserving manufacturing capacity for future pandemics
}

\author{
Emma Hannay, ${ }^{1}$ Marta Fernández-Suárez, ${ }^{1}$ Philippe Duneton ${ }^{2}$
}

To cite: Hannay E,

Fernández-Suárez M, Duneton $\mathrm{P}$. COVID-19 diagnostics: preserving manufacturing capacity for future pandemics. BMJ Global Health

2022;7:e007494. doi:10.1136/ bmjgh-2021-007494

Handling editor Seye Abimbola

Received 4 October 2021 Accepted 27 January 2022
Check for updates

(c) Author(s) (or their employer(s)) 2022. Re-use permitted under CC BY-NC. No commercial re-use. See rights and permissions. Published by BMJ.

${ }^{1}$ FIND, Geneva, Switzerland ${ }^{2}$ Unitaid, Geneva, Switzerland

Correspondence to Dr Emma Hannay; Emma.Hannay@finddx.org

\section{INTRODUCTION}

Manufacturing capacity challenges with COVID-19 vaccines have recently hit the headlines, ${ }^{1}$ however, capacity deficits have been a limiting factor across commodities since the pandemic began. During the first half of 2020, a huge surge in demand for personal protective equipment led to acute supply restrictions, ${ }^{2}$ and shortages of reagents used in molecular diagnostics, such as RNA extraction kits and lysis buffers, severely limited testing capacities. ${ }^{3}$

Capacity limitations to the manufacture of COVID-19 rapid diagnostic tests (RDTs) have also caused supply constraints. The speed at which COVID-19 RDTs were developed and the subsequent surge in use of antigen RDTs in public health settings have necessitated extensive effort and investment to meet volume demands. As RDT manufacture can be switched from one disease to another with relative ease (although with a trade-off in efficiency), the resulting increase in RDT manufacturing capacity should enable a more rapid response to future threats. However, concerns have been raised that this capacity could be lost once the COVID-19 pandemic is over.

\section{IMPORTANCE OF RDT MANUFACTURING CAPACITY}

Diagnostics are an essential part of sustainable, resilient health systems and pandemic preparedness plans. ${ }^{4}$ For optimal impact, testing needs to be widespread. As the cost and complexity of molecular tests prohibits their use in many areas, RDTs-which are inexpensive, simple to use and can be performed at the point of care-are crucial to the success of outbreak responses.

In an outbreak situation, RDTs need to be produced and distributed quickly in huge volumes, which requires manufacturing capacity to be in place before the outbreak begins. Unfortunately, prior to COVID-19,
Summary box

Manufacturing capacity for COVID-19 antigen rapid diagnostic tests is estimated to have increased by $200 \%$ since the pandemic began, with further increases anticipated over the coming year.

- Manufacturers reported numerous challenges to scale up, including need for improved manufacturing techniques, new equipment and plants, difficulties forecasting demand and supply shortages of raw materials.

- As supply will exceed demand after the pandemic is over, strategies to preserve sufficient production capacity for future pandemics must be implemented.

- Proposed strategies include expansion of local manufacturing capacity for tests and/or their components, creation of sustainable demand, incentives to maintain dormant equipment, investment in surveillance and stockpiling, and diversification of supply chains for critical components.

this was not the case, thus manufacturers found themselves rushing to fill a capacity gap.

\section{CHALLENGES TO MANUFACTURING CAPACITY SCALE-UP}

To explore the challenges to scale up of production capacity to meet the huge demand for COVID-19 RDTs, FIND, the global alliance for diagnostics, sent a written questionnaire to 29 manufacturers of COVID-19 RDTs, including the 20 companies with current highest production capacity (based on volume produced between August 2020 and January 2021). In total, 17 manufacturers responded. Although the sample is not fully representative of all RDT manufacturers, it includes 13 manufacturers from South Korea and China, the most active countries in production of COVID-19 RDTs, and those with and without stringent regulatory authority approvals for their products. 
Respondents noted that production lines for existing RDTs were inefficient and production equipment was insufficient to meet the high demand. New automated assembly lines and improved manufacturing techniques had to be developed, new machinery and production plants purchased, and new staff recruited and trained. Manufacturers also reported difficulties in forecasting demand for COVID-19 RDTs, leading to uncertainty over how much could be realistically invested in scale-up activities.

Supply of raw materials and kit materials common to many RDTs, such as nitrocellulose membranes, sterile swabs, plastic cassettes and test reagents, was a limiting factor in 2020 when RDTs were first developed, due to the small number of suppliers and to long and fragile international supply chains. These issues have to some extent been addressed, as suppliers have implemented their own scale-up initiatives, but six of the survey respondents reported concerns around increasing prices of certain raw materials and the ongoing impact of this on COVID-19 RDT production capacity.

\section{MAGNITUDE OF MANUFACTURING CAPACITY INCREASES}

Despite the challenges to scale up, COVID-19 RDT manufacturers have been able to substantially increase production capacity since the pandemic began. Based on the survey responses, overall production capacity is estimated to have doubled in 7 months, from 482 million tests per month in October 2020 to 1006 million in April 2021 (figure 1). RDTs with WHO Emergency Use Listing (EUL) or RDTs under WHO EUL assessment represent approximately $50 \%$ of the total shown for antigen RDTs.

The increase in manufacturing capacity was primarily driven by antigen RDTs, for which capacity increased by $200 \%$, and was accompanied by a $30 \%$ decrease in the average price per test, according to eight manufacturers who provided cost details. Manufacturers predict further growth over the next year, reaching a potential 1.9 billion tests per month in 2022.



Figure 1 Estimated increase in combined COVID-19 RDT production capacity from 17 manufacturers (predicted number of tests per month if facilities are run at maximum (millions)). RDTs, rapid diagnostic tests.
HOW MANUFACTURING CAPACITY INCREASES COULD BE LOST

Due to the increase in production capacity for RDTs, supply is predicted to exceed demand after the acute spike of COVID-19 is over. Without economic incentives to keep the additional manufacturing equipment and plants running and staff employed, many of the gains made during COVID-19 are likely to be lost. Feedback from manufacturers suggests that while new production plants may not be decommissioned post-pandemic, it will not be financially viable to maintain the human resources required to keep these additional plants running.

Furthermore, the rapid increase in RDT production volume has caused manufacturing capacity for RDTs to become geographically concentrated, as capacity building occurred in silos without coordination across manufacturers. As such, sustainability of production capacity postpandemic may be low, while fragility, in terms of potential impact of outbreaks and subsequent border closures in the countries where manufacturers are based, is high.

\section{STRATEGIES TO PRESERVE MANUFACTURING CAPACITY}

While post-COVID-19 capacity may be surplus to requirements, we must ensure that sufficient capacity is preserved to strengthen pandemic preparedness for the future. Creation of sustainable demand will be a central part of any strategy (figure 2). Establishing volume guarantees (ie, minimal viable volumes to break even) postpandemic, by building demand for RDTs for surveillance of other diseases with pandemic potential, would provide incentive to maintain some of the capacity increases developed for COVID-19 RDTs. However, any demandbuilding efforts must incorporate a quality element, as low-quality RDTs can have a negative impact on disease management and public confidence.

Provision of funding to stockpile RDTs during nonpandemic periods, using surveillance needs to rotate stockpiles, would add to this incentive. But this would require substantial investment in research and development. Currently, there are no diagnostics available for $60 \%$ of the WHO R\&D Blueprint diseases. ${ }^{4}$ Use of surplus capacity to improve availability of RDTs for primary care screening programmes, such as human papillomavirus testing for women's health, may help to fill the gap.

Governments should work with RDT manufacturers to find incentives to maintain dormant equipment during non-pandemic periods, for example, via subsidies to produce RDTs for diseases of regional public health significance. The international Pandemic Preparedness Partnership has set a target for deployment of vaccines within 100 days of future infectious disease outbreaks. ${ }^{5}$ Maintenance of RDT manufacturing equipment would help to attain the same targets for diagnostics. Development of flexible, innovative and sustainable manufacturing equipment and processes could also support rapid switching of manufacturing lines to emergency mass production during outbreaks. 




Figure 2 Strategies to preserve diagnostic manufacturing capacity.

RDT manufacturing capacity should be diversified, through expansion of local manufacturing capacities to improve sustainability of production capacity and to maintain distributed capacity across regions. Building out of manufacturing hubs for RDTs and/or their less complex components in low/middle-income countries is recommended to create affordability and local ownership, particularly as shipping costs for finished products or import of raw materials can impact overall RDT costs.

Finally, diversification of supply chains for nonspecialised critical components such as the plastic cassette, sterile swabs and nitrocellulose membrane will be important to prevent the bottlenecks caused by raw material shortages seen during the current pandemic, and to introduce competition to ensure that the price of raw materials is not prohibitively high.

\section{CONCLUSIONS}

Failure to preserve sufficient RDT production capacity after the COVID-19 pandemic will lead to a repetition of the scale-up activities undertaken to address COVID-19 for future outbreaks. Preservation will protect the significant financial investments that have already been made, and potentially allow unit cost of tests for future outbreaks to be reduced, as manufacturers will not need to invest heavily in scale-up.

The strategies proposed were developed with RDTs in mind, however, there is some degree of extrapolation to molecular tests, which can also be impacted by shortages of materials such as swabs and reagents. Some learning may also be applicable to vaccines, therapeutics and other commodities.

Preservation of manufacturing capacity is just one component of an overall strategy to improve diagnostic pandemic preparedness. In order to rapidly develop and deploy new RDTs for future outbreaks, manufacturers will also require support with other elements of the development process such as clinical validation of new tests and navigation of complex country-level registration procedures.

The impact of low manufacturing capacity for diagnostics in outbreak situations was predicted long before the emergence of COVID- $19,{ }^{4}$ but it took a global pandemic to generate the changes needed. We must capitalise on the improvements brought about by the response to COVID-19 to ensure that the progress we have made is not squandered.

Acknowledgements The production capacity analysis was led by Kavi Ramjeet at FIND. Medical writing services, funded by FIND, were provided by Rachel Wright, in accordance with Good Publication Practice (GPP3).

Contributors All three authors have contributed jointly to the structuring and drafting of this article.

Funding This work arose from FIND's efforts to understand the COVID-19 diagnostics market as part of wider work in increasing access to COVID-19 diagnostic testing that has been supported by a range of bilateral and multilateral funders of the Access to COVID-19 Tools Accelerator (ACT-Accelerator) Diagnostics Pillar. Data collection for the survey of manufacturers was funded directly by a grant from the UK Foreign, Commonwealth and Development Office (UK FCD0; grant/award number: not applicable).

Competing interests On behalf of FIND, EH provides leadership to the ACTAccelerator Diagnostics Pillar, a partnership launched by the WHO and the European Commission to increase access to diagnostic testing. EH and MF-S are employees of FIND. PD is an employee of Unitaid. All authors had full access to all the data included in this article and accept responsibility to submit for publication.

\section{Patient consent for publication Not required}

Ethics approval This study does not involve human participants.

Provenance and peer review Not commissioned; externally peer reviewed.

Data availability statement Data are available upon request.

Open access This is an open access article distributed in accordance with the Creative Commons Attribution Non Commercial (CC BY-NC 4.0) license, which permits others to distribute, remix, adapt, build upon this work non-commercially, and license their derivative works on different terms, provided the original work is properly cited, appropriate credit is given, any changes made indicated, and the use is non-commercial. See: http://creativecommons.org/licenses/by-nc/4.0/.

\section{REFERENCES}

1 Irwin A. What it will take to vaccinate the world against COVID-19. Nature 2021;592:176-8.

2 Burki T. Global shortage of personal protective equipment. Lancet Infect Dis 2020;20:785-6.

3 Esbin MN, Whitney ON, Chong S, et al. Overcoming the bottleneck to widespread testing: a rapid review of nucleic acid testing approaches for COVID-19 detection. RNA 2020;26:771-83.

4 Kelly-Cirino CD, Nkengasong J, Kettler $\mathrm{H}$, et al. Importance of diagnostics in epidemic and pandemic preparedness. BMJ Glob Health 2019;4:e001179.

5 Department of Health and Social Care. New global partnershop launched to fight future pandemics, 2021. Available: https://www. gov.uk/government/news/new-global-partnership-launched-to-fightfuture-pandemics [Accessed 11 Jun 2021]. 